Article

\title{
Evaluation of the Impact of Different Pain Medication and Proton Pump Inhibitors on the Osteogenic Differentiation Potential of hMSCs Using ${ }^{99 m}$ Tc-HDP Labelling
}

\author{
Tobias Grossner $1, * \mathbb{C}$, Uwe Haberkorn ${ }^{2,3,4}$ and Tobias Gotterbarm ${ }^{5}$ \\ 1 Trauma Surgery and Paraplegiology, Clinic for Orthopedics and Trauma Surgery, Center for Orthopedics, \\ University Hospital Heidelberg, 69120 Heidelberg, Germany \\ 2 Department of Nuclear Medicine, University Hospital Heidelberg, 69120 Heidelberg, Germany; \\ uwe.haberkorn@med.uni-heidelberg.de \\ 3 Clinical Cooperation Unit Nuclear Medicine, Deutsches Krebsforschungszentrum (DKFZ), \\ 69120 Heidelberg, Germany \\ 4 Translational Lung Research Center Heidelberg (TLRC), German Center for Lung Research (DZL), \\ 69120 Heidelberg, Germany \\ 5 Department of Orthopedics and Traumatology, Kepler University Hospital, 4020 Linz, Austria; \\ tobias.gotterbarm@kepleruniklinikum.at \\ * Correspondence: tobias.grossner@med.uni-heidelberg.de; Tel.: +49-6221-56-35-443
}

Citation: Grossner, T.; Haberkorn, U.; Gotterbarm, T. Evaluation of the Impact of Different Pain Medication and Proton Pump Inhibitors on the Osteogenic Differentiation Potential of hMSCs Using ${ }^{99 \mathrm{~m}} \mathrm{Tc}-\mathrm{HDP}$ Labelling. Life 2021, 11, 339. https://doi.org/ $10.3390 /$ life11040339

Academic Editor: Christopher Lai

Received: 4 March 2021

Accepted: 9 April 2021

Published: 11 April 2021

Publisher's Note: MDPI stays neutral with regard to jurisdictional claims in published maps and institutional affiliations.

Copyright: (c) 2021 by the authors. Licensee MDPI, Basel, Switzerland. This article is an open access article distributed under the terms and conditions of the Creative Commons Attribution (CC BY) license (https:// creativecommons.org/licenses/by/ $4.0 /)$.

\begin{abstract}
First-line analgetic medication used in the field of musculoskeletal degenerative diseases, like Nonsteroidal anti-inflammatory drugs (NSAIDs), reduces pain and prostaglandin synthesis, whereby peptic ulcers are a severe adverse effect. Therefore, proton pump inhibitors (PPI) are frequently used as a concomitant medication to reduce this risk. However, the impact of NSAIDs or metamizole, in combination with PPIs, on bone metabolism is still unclear. Therefore, human mesenchymal stem cells (hMSCs) were cultured in monolayer cultures in 10 different groups for 21 days. New bone formation was induced as follows: Group 1 negative control group, group 2 osteogenic differentiation media (OSM), group 3 OSM with pantoprazole (PAN), group 4 OSM with ibuprofen (IBU), group 5 OSM with diclofenac (DIC), group 6 OSM with metamizole (MET), group 7 OSM with ibuprofen and pantoprazole (IBU + PAN), group 8 OSM with diclofenac and pantoprazole (DIC + PAN), group 9 OSM with metamizole and pantoprazole (MET + PAN) and group 10 OSM with diclofenac, metamizole and pantoprazole (DIC + MET + PAN). Hydroxyapatite content was evaluated using high-sensitive radioactive ${ }^{99 \mathrm{~m}} \mathrm{Tc}-\mathrm{HDP}$ labeling. Within this study, no evidence was found that the common analgetic medication, using NSAIDs alone or in combination with pantoprazole and/or metamizole, has any negative impact on the osteogenic differentiation of mesenchymal stem cells in vitro. To the contrary, the statistical results indicate that pantoprazole alone (group 3 (PAN) $(p=0.016)$ ) or diclofenac alone (group 5 (DIC) $(p=0.008)$ ) enhances the deposition of minerals by hMSCS in vitro. There is an ongoing discussion between clinicians in the field of orthopaedics and traumatology as to whether post-surgical (pain) medication has a negative impact on bone healing. This is the first hMSC in vitro study that investigates the effects of pain medication in combination with PPIs on bone metabolism. Our in vitro data indicates that the assumed negative impact on bone metabolism is subsidiary. These findings substantiate the thesis that, in clinical medicine, the patient can receive every pain medication needed, whether or not in combination with PPIs, without any negative effects for the osteo-regenerative potential.
\end{abstract}

Keywords: mesenchymal stem cells; osteogenic differentiation; proton pump inhibitors; NSASIDs; ${ }^{99 \mathrm{~m}} \mathrm{Tc}-\mathrm{HDP}$ labeling; hydroxyapatite; metamizole

\section{Introduction}

In an aging population, the impact of musculoskeletal degenerative diseases (e.g., arthrosis and osteoporosis) and trauma is an enormous burden for healthcare systems [1]. 
At a certain point of treatment, nearly all patients who consult a doctor or physiotherapist due to a musculosceletal disorder are prescribed pain medication $[2,3]$.

Usually, the pain medication strategy follows recommendations in guidelines published by large national societies like the "American Pain Society" [4] or the "Agreement of the Professional Association of German Anesthesiologists and the Professional Association of German Surgeons for the Organization of Postoperative Pain Therapy" [5] or the -S3guideline for long-term application for opioids (LONTS) [6]. Interestingly, the WHO "Web statement on pain management guidance" states that the WHO is currently reviewing and updating its guidelines and policy documents regarding pain management.

Non-opioid drugs are the backbone of any adequate pain medication concept. Most of these drugs are NSAIDs (nonsteroidal anti-inflammatory drugs). Examples include acetylsalicylic acid (e.g., Aspirin ${ }^{\circledR}$ ) (Bayer AG, Monheim am Rhein, Germany), ibuprofen (e.g. Nurofen ${ }^{\circledR}$ ) (Reckitt Benckiser Deutschland $\mathrm{GmbH}$, Heidelberg, Germany) and diclofenac (e.g., Voltaren ${ }^{\circledR}$ ) (GlaxoSmithKline Consumer Healthcare GmbH \& Co. KG, Munich, Germany) [2]. The anti-inflammatory potential of these drugs reduces symptoms caused by the inflammatory component in pathologies like activated arthrosis and post-surgical inflammatory response [7]. An additional feature of NSAIDs (e.g., diclofenac and ibuprofen) is their ability to reduce heterotopic ossifications after total hip arthroplasty and acetabular fracture treatment [8-12]. Non-selective NSAIDs inhibit COX-1 and COX-2, while selective NSAIDs only inhibit COX-2. Both pathways suppress the synthesis of prostaglandins, an important mediator for inflammation. This suppression upregulates the production of gastric acid [13] which causes one of the most severe side effects of these drugs, namely gastrointestinal ulcers. Proton pump inhibitors (PPIs) are therefore frequently used as an accompanying medication during treatment with NSAIDs to prevent such gastrointestinal side effects [13]. However, non-NSAIDs are also widely used for first-line pain medication. Here, metamizole (e.g., Novalgin ${ }^{\circledR}$ ) (Sanofi-Aventis Deutschland GmbH, Frankfurt am Main, Germany) and paracetamol (e.g., Tylenol ${ }^{\circledR}$ ) (Johnson \& Johnson Consumer Inc, Fort Washington, PA 19034, USA) are the most common agents. While their analgetic potential is at least as high as NSAIDs, their anti-inflammatory potential is comparably less [3,7].

The potential relationship between PPIs and fracture risk is disputed and the recent data analysis from the Trøndelag Health Study (HUNT) study did not reveal any fracture risk associated with the use of PPIs $[14,15]$. While little in vitro data is available on the impact of PPIs on osteoblast and osteoclast-like cells, some pilot projects assume a negative impact of PPIs on bone metabolism [16], while other studies show no negative effects on primary human osteoblasts [17]. There are indications that bone degradation and resorption are inhibited by PPIs due to reduced osteoclast activity [18]. Data about the impact of NSAIDs on bone metabolism and bone healing are inconclusive. Recent systematic reviews found no consensus regarding the risks and benefits of treating musculoskeletal disorder patients with NSAIDs and the impact of these drugs on bone metabolism [19], while another review assumes the absence of robust clinical and scientific data [20]. There is also a lack of data concerning the effects of non-NSAIDs like metamizole on bone metabolism and no data available concerning the effects of metamizole in combination with NSAIDs and PPIs. Furthermore, no experimental data is available for evaluating the effects of standard pain medication (NSAIDS and non-NSAIDs) in combination with PPI therapy on the osteogenic potential of human mesenchymal stem cells in vitro.

Recently, ${ }^{99 \mathrm{~m}} \mathrm{Tc}-\mathrm{HDP}$ labeling has been employed as a highly sensitive, non-destructive and effective method to quantify the amount of hydroxyapatite within osteogenic-induced monolayer cell cultures of human mesenchymal stem cells This method has been positively verified by correlation analysis with the standard marker for osteogenesis in vitro in previous studies (quantitative Alizarin red staining, von Kossa staining, scanning electron microscope with energy-dispersive $\mathrm{X}$-ray spectroscopy, inductively coupled plasma mass spectrometry for absolute calcium) [21]. Here, the radioactive tracer ${ }^{99} \mathrm{~m}$ Tc bound to a polyphosphonate binds to inorganic mineral in areas with high osteogenic activity, e.g., hydroxyapatite deposition, by chemisorption. As ${ }^{99 \mathrm{~m}}$ Technetium decays by gamma 
decay, emitting energy with $140 \mathrm{keV}$, these counts can be easily detected and registered with a gamma camera. The amount of bound tracer is proportional to the amount of hydroxyapatite deposition and to the osteogenic potential of the experimental cell culture setting [21].

This experimental in vitro study, therefore, seeks to discover the effect of clinically used pain medication on new bone formation by human mesenchymal stem cells (hMSCs) to evaluate if single and combined non-opioid analgetic drugs exert any impact on the osteogenic response of human mesenchymal stem cells and if the accompanying PPI medication exert any influence on this impact.

\section{Results}

\section{1. ${ }^{99 m}$ Tc-HDP Labelling}

The human donors exhibited marginal variability regarding individual osteogenic potential for the uptake of the tracer within each group. The negative control group (group 1, NCG) showed almost no uptake (mean 327 counts/180 s) while the highest mean uptake was seen within group 5 treated with diclofenac (mean 61,047 counts/180 s). The second highest uptake was discovered for the group treated with pantoprazole (group 3, mean 60,333 counts/180 s), followed by group 4 treated with ibuprofen (mean 59,124 counts $/ 180 \mathrm{~s}$ ). The mean uptake within the other groups was between 55,939 and 58,624 counts $/ 180 \mathrm{~s}$.

The tracer uptake was at least 171-fold higher within the osteogenic groups (groups 2-10) compared to the negative control group (group 1) with the lowest uptake (group 10, DIC + MET + PAN) 55,939 counts/180 s), while it was 186-fold higher for the osteogenic group with the highest uptake (group 5 DIC, 61,047 counts /180 s).

A complete overview regarding the tracer uptake is shown in Table 1.

Table 1. Mean, minimum and maximum gamma counts/180 s for each group.

\begin{tabular}{cccccc}
\hline Group & N & Mean & Minimum & Maximum & $\begin{array}{c}\text { Standard } \\
\text { Deviation }\end{array}$ \\
\hline Group_1_NCG & 12 & 328 & 2 & 1170 & 322 \\
\hline Group_2_OSM & 12 & 54,880 & 46,194 & 60,107 & 5061 \\
\hline Group_3_PAN & 12 & 60,333 & 46,627 & 64,402 & 4791 \\
\hline Group_4_IBU & 12 & 59,124 & 49,896 & 63,246 & 4403 \\
\hline Group_5_DIC & 12 & 61,048 & 51,602 & 65,606 & 3939 \\
\hline Group_6_MET & 12 & 56,246 & 45,070 & 60,775 & 5159 \\
\hline Group_7_IBU_PAN & 12 & 58,624 & 50,548 & 62,402 & 3859 \\
\hline Group_8_DIC_PAN & 12 & 56,694 & 43,372 & 64,111 & 5349 \\
\hline Group_9_MET_PAN & 12 & 56,778 & 50,133 & 60,326 & 2972 \\
\hline Group_10_DIC_MET_PAN & 12 & 55,940 & 47,865 & 61,387 & 4562 \\
\hline
\end{tabular}

\subsection{Statistical Results}

The Kolmogorov-Smirnov test for normal distribution was performed prior to further data analysis and revealed a normal distribution for tracer uptake within each of the ten groups $(p \leq 0.001)$.

The first Analysis of variance (ANOVA) analysis revealed a highly significant effect of the osteogenic media for all osteogenic groups $(2,3,4$ and 7$)$ versus the negative control group (group 1) with $p \leq 0.001$, revealing a successful osteogenic differentiation. Additionally, a significantly higher uptake was noted for the group where only pantoprazole (group 3 ) was added versus the group in which only the standard osteogenic media (group 2) was used ( $p \leq 0.05$ ). No significantly higher uptake was noted for the group in which IBU (group 4) and IBU + PAN (group 7) was added ( $p=0.135$ and $p=0.284$ ) versus the 
standard osteogenic group (group 2). Therefore, no significant negative effect regarding the osteogenesis could be shown by adding ibuprofen or ibuprofen and pantoprazole to the cell culture media, while adding just pantoprazole alone resulted in a significant enhancement of the tracer uptake.

The second ANOVA analysis also revealed a highly significant effect of the osteogenic media for all osteogenic groups $(2,3,5$ and 8$)$ versus the negative control group (group 1) with $p \leq 0.001$, and so also revealed successful osteogenic differentiation. Additionally, a significantly higher uptake was noted for the group where only pantoprazole (group 3) was added versus the group in which only the standard osteogenic media (group 2) was used $(p \leq 0.05)$. Additionally, there was a significantly higher uptake $(p \leq 0.01)$ of the tracer in group 5. Here, only diclofenac was added to the standard osteogenic media (group 2). No significantly higher uptake was noted for the group in which DIC + PAN (group 8) was added $(p=1.00)$ versus the standard osteogenic group (group 2$)$. Therefore, a significant effect regarding osteogenesis could be shown by adding pantoprazole or diclofenac to the cell culture media, while adding diclofenac and pantoprazole did not result in a significant enhancement of the tracer uptake.

The third ANOVA analysis also revealed a highly significant effect of the osteogenic media for all osteogenic groups (2, 3, 6 and 9) versus the negative control group (group 1) with $p \leq 0.001$, which also revealed a successful osteogenic differentiation under these conditions. Additionally, a significantly higher uptake was noted for the group where only pantoprazole (group 3) was added versus the group in which only the standard osteogenic media (group 2 ) was used $(p \leq 0.05)$. No significantly higher uptake was noted for the groups in which MET (group 6) and MET + PAN (group 9) were added ( $p=0.925$ and $p=0.998$ ) versus the standard osteogenic group (group 2). Therefore, no significant effect regarding osteogenesis could be shown by adding either metamizole or metamizole and pantoprazole to the culture media, whereas adding just pantoprazole led to a significant enhancement of the tracer uptake.

Likewise, the fourth ANOVA analysis revealed a highly significant effect of the osteogenic media for all osteogenic groups (2,3 and 10) versus the negative control group (group 1 ) with $p \leq 0.001$. Additionally, in this analysis, a significantly higher uptake was noted for the group where only pantoprazole (group 3 ) was added versus the group in which only the standard osteogenic media (group 2$)$ was used $(p=0.016)$, while no significant effect was shown by adding a combination of DIC + MET + PAN (group 10) to the cell culture media compared to the standard osteogenic media (group 2) $(p=1)$.

In summary, no significant effect regarding osteogenesis could be shown by adding metamizole in combination with diclofenac and pantoprazole to the cell culture media, while adding just pantoprazole led to a significant enhancement of the tracer uptake.

The mean values of each group with the corresponding ANOVA analyses 1-4 are shown in Figures 1-4. 


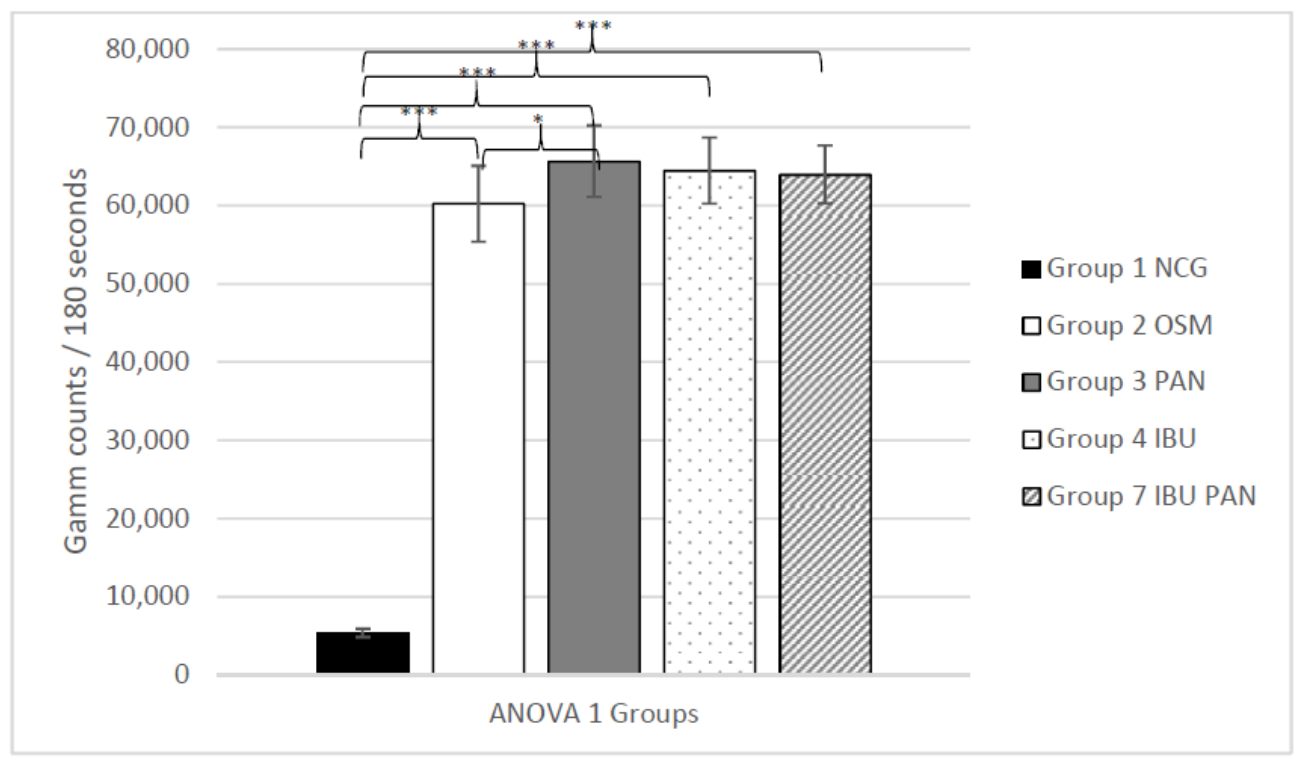

Figure 1. ANOVA 1, analyzing the ${ }^{99 \mathrm{~m}} \mathrm{TC}-\mathrm{HDP}$ uptake (counts) $/ 180 \mathrm{~s}$ for groups $1-4$ and $7+/-$ standard deviation. Statistical significance is indicated by ${ }^{* *}=p \leq 0.001 ;{ }^{*}=p \leq 0.05$.

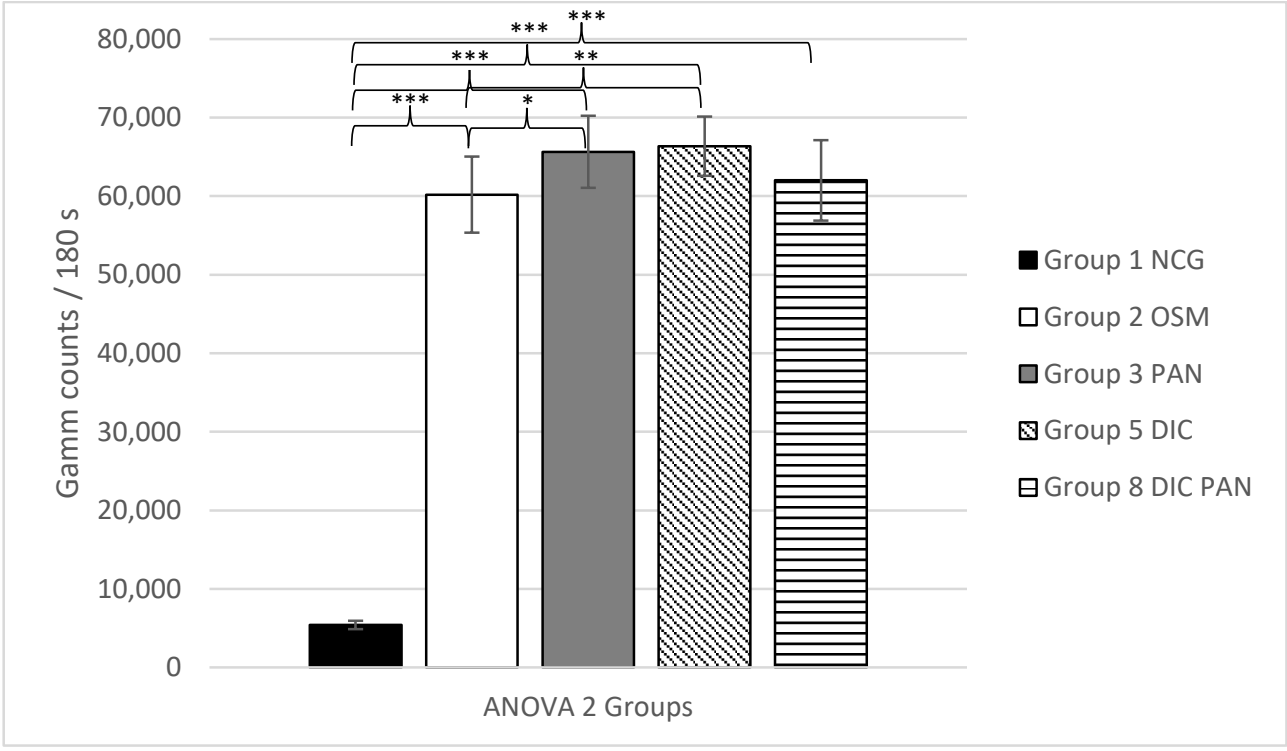

Figure 2. ANOVA 2, analyzing the ${ }^{99 \mathrm{~m}} \mathrm{TC}-\mathrm{HDP}$ uptake (counts) $/ 180 \mathrm{~s}$ for groups $1-3,5$ and $8+/-$ standard deviation. Statistical significance is indicated by ${ }^{* *}=p \leq 0.001{ }^{* *}=p \leq 0.01,^{*}=p \leq 0.05$. 


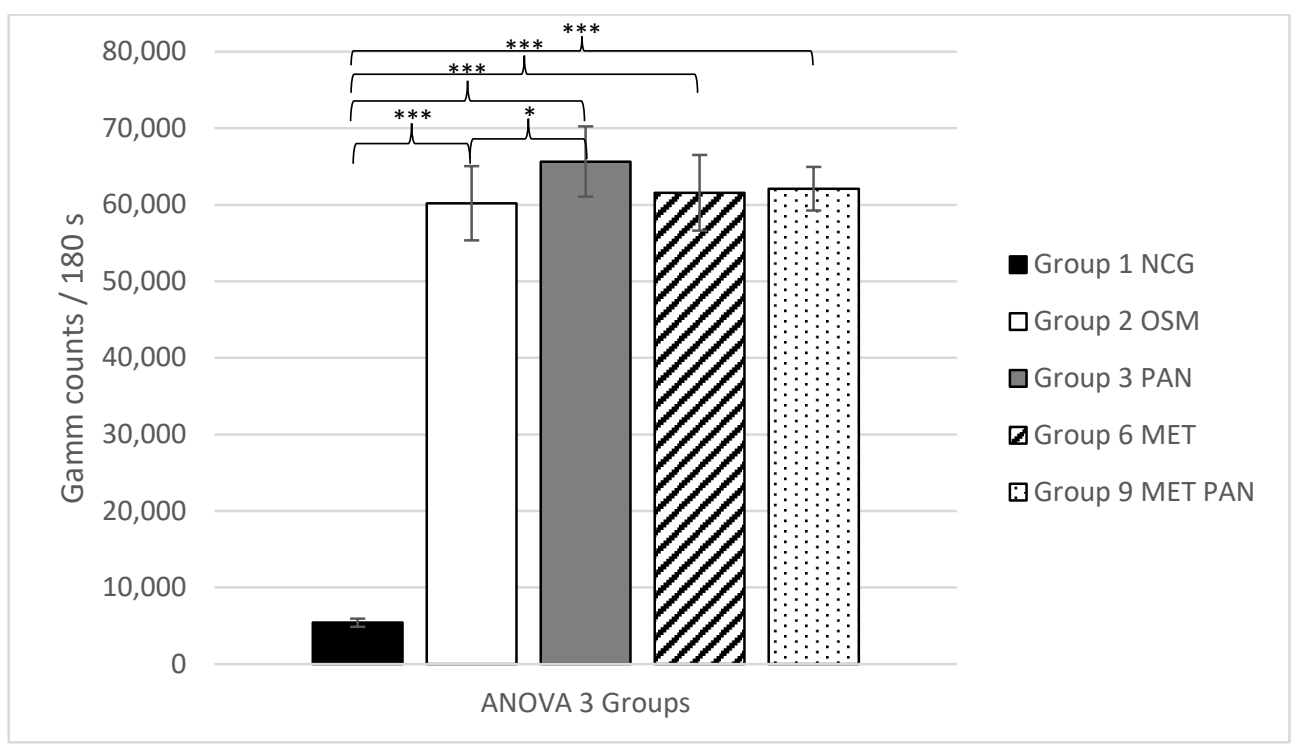

Figure 3. ANOVA 3, analyzing the ${ }^{99} \mathrm{~m}$ TC-HDP uptake (counts) $/ 180 \mathrm{~s}$ for groups $1-3,6$ and $9+/-$ standard deviation. Statistical significance is indicated by ${ }^{* *}=p \leq 0.001 ;{ }^{*}=p \leq 0.05$.

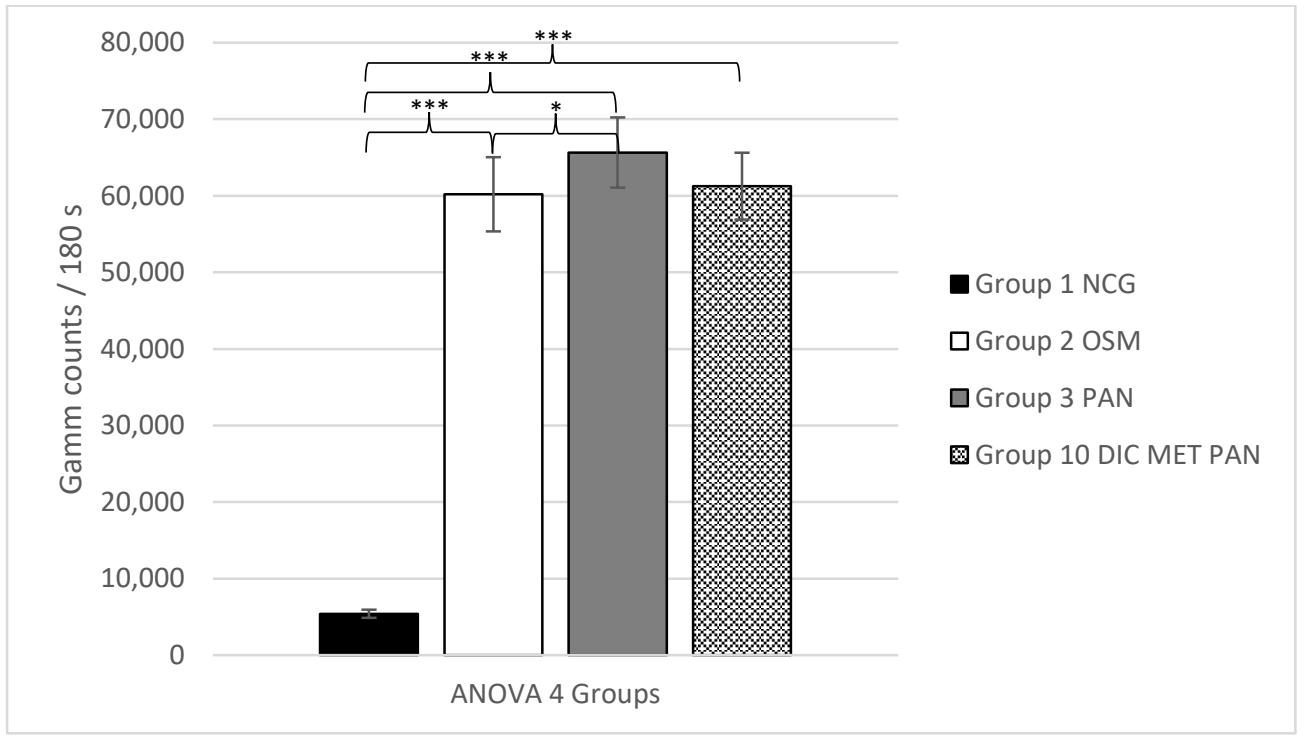

Figure 4. ANOVA 4, analyzing the ${ }^{99 \mathrm{~m}} \mathrm{TC}-\mathrm{HDP}$ uptake (counts) $/ 180 \mathrm{~s}$ for groups $1-3$ and $10+/-$ standard deviation. Statistical significance is indicated by ${ }^{* *}=p \leq 0.001 ;{ }^{*}=p \leq 0.05$.

\section{Discussion}

Musculoskeletal disorders require sufficient pain medication for a symptomatic treatment [2]. If a surgical treatment is necessary, whether this is an elective orthopaedic procedure (e.g., total hip replacement due to progredient arthrosis) or a trauma surgical emergency procedure (e.g., reduction and osteosynthesis due to a fracture), it is critical that, in the post-surgical setting, the patient is almost pain free to allow fast recovery, reduce morbidity and mortality, to avoid discomfort and to ensure immediate mobilization $[4,7]$. However, recent evidence indicates that certain (pain) medication may have an unknown effect on bone metabolism [20].

The most important finding of our study is that the NSAIDs tested, as well as the non-NSAID metamizole, do not have a negative effect on the hydroxyapatite synthesis of human MSCs, the key marker of osteogenesis, under in vitro conditions and so do not affect the osteogenic differentiation. While this study focused on the hydroxyapatite production by the hMSCs within the different groups, no direct evaluation of the potential 
impact of the drugs on the viability of the cells was performed. If any of the drugs or their combination caused a critical impact on the cell viability, a significantly lower production of hydroxyapatite would have been expected. However, the analysis of the mean uptake values of all groups (3-8) where one or more drugs were added showed a higher uptake of the tracer compared to group (2) where only the osteogenic supplements were added. Therefore, it can be assumed that none of the drugs affected the cell viability substantially. In all the ANOVA analyses performed, there was a highly significantly major uptake of the radioactive tracer $(p \leq 0.001)$ within all groups treated with OSM enriched with ibuprofen (group 4), diclofenac (group 5) and metamizole (group 6), as compared to the negative control group (group 1). These results concur with the results from previous in vitro studies that also revealed no negative effect on the osteogenic differentiation [22,23]. Normal levels of ibuprofen seem to have no impact on the osteogenic response of MSCs in vitro [22], while other data indicates that the osteogenic potential of MSCs is inhibited/delayed by the treatment of high-dose NSAIDs under inflammatory conditions [24]. Another study assumes a potential reduction in bone metabolism, as their data indicate an inhibition of the chondrogenesis that might affect enchondral bone formation by NSAIDs [23]. Based on this theory, there are some recommendations that high-risk patients should not be treated with NSAIDs [20]. As our in vitro model does not support enchondral bone formation, no statement can be made from our results regarding this thesis. Other in vitro studies indicate that the osteogenic marker alkaline phosphatase, bone sialoprotein, and osteocalcin, and chondrogenesis marker gene aggrecan can be negatively affected by certain pain drugs while diclofenac is the only NSAID that has no negative effect upon all of these osteogenic markers [25]. Our analysis showed not only that diclofenac has no negative effect upon osteogenesis, but also a significant positive impact on the in vitro osteogenesis, resulting in a clearly higher uptake $(p \leq 0.05)$ of the tracer in the group additionally treated with diclofenac (group 5) compared to the standard osteogenic group (group 2), even in our multi donor in vitro setting. This result is surprising from a clinical point of view, as diclofenac, as well as other NSAIDs, is known to suppress osteogenesis and reduce heterotopic ossifications $[9,10]$. Other in vitro data support our results, as it has been shown that diclofenac is the only NSAID that does not negatively affect the expression of the osteogenic key markers in MSCs [25]. Thus, there is evidence, supported by our results, that diclofenac supports in vitro osteogenic differentiation but the mechanism remains unknown. It is known, that BM-MSCs have compensatory mechanism to restore PEG2 synthesis as an intrinsic regulation of osteogenic differentiation if these cells are treated with NSAIDs [26] while the inhibition of the prostaglandin synthesis in vivo result is a limitation of pathologic bone growth, which makes these drugs very popular to avoid heterotopic ossifications after total hip replacement $[10,11]$. However, recent systematic reviews confirmed the absence of robust in vitro or clinical data regarding NSAIDs and their impact on bone formation, and encouraged researchers to perform further basic science and clinical studies to clarify the risks and benefits of NSAIDs in patients with musculoskeletal disorders [19]. Unfortunately, the direct hydroxyapatite content has never been evaluated, neither in the studies investigating in the effects of NSAID nor in the studies investigating in the effects of PPI upon the osteogenic differentiation in vitro. We define the absolute hydroxyapatite content as one of the most important parameters that should always be quantified directly in all in vitro studies regarding the effects on bone metabolism in vitro as it is a functional and direct assessment of the mineral. Previous in vitro data regarding the effect of PPIs on osteogenesis showed a negative impact, resulting in a deleterious effect on bone cells $[16,27,28]$, while our data distinctly indicates a consistently beneficial effect on the osteogenesis by adding just PPIs to the cell cultures of MSCs $(p \leq 0.05)$. This might be a result of increased mitochondrial activity, as previously shown [17]. However, no significant positive effect on the osteogenesis could be shown by adding PPIs as a concomitant medication to the drugs ibuprofen, diclofenac or metamizole. It has to be noted that, within our experimental set-up, we investigated the PPI impact on in vitro osteogenesis by using only one PPI (Pantoprazole). Beside Pantoprazole, there various other PPIs available 
(Omeprazole, Esomeprazole, Dexlansoprazole, etc.) that all act by irreversibly blocking the hydrogen/potassium adenosine triphosphatase enzyme. Regarding their configuration PPIs can be generally divided into the benzimidazole group or the imidazopyridine, while all commercially available PPIs belong to the benzimidazole group. Nevertheless, as all of these drugs have a slightly different chemic structure, it cannot be assumed that the presented results can be generalized for all PPIs. Therefore, additional studies will be necessary to compare the different PPIs and their effect upon osteogenic differentiation in vitro.

Additionally, combining diclofenac and pantoprazole has no further positive effect on osteogenesis $(p=1)$, even though both drugs individually have a significant positive impact $(p \leq 0.05, p \leq 0.01)$. The combination of diclofenac, metamizole and pantoprazole has no beneficial effect on the osteogenesis of hMSCs as well.

In summary, our in vitro data suggest no negative effect of the NSAIDs ibuprofen and diclofenac or the non-NSAID metamizole on the osteogenesis of MSCs, while the NSAID diclofenac and the PPI pantoprazole enhance the production of hydroxyapatite. No negative effect of any single drug or combination of drugs on bone metabolism in vitro could be revealed. Further, no additional positive effect was found by combining the pain-reliving drugs with the PPI pantoprazole.

As no basic research or clinical data is available concerning the effects of different NSAIDs and other pain drugs in combination with PPIs towards bone metabolism, especially not in the context of evaluating the hydroxyapatite content, there is a strong need to perform further studies in this field, as it is very important to treat the aging population with appropriate pain medication without the risk of negatively influencing their bone metabolism.

Limitation of the study: We use a standard monolayer cell culture model, so it has to be kept in mind that the physiological pathways in humans are far more complex and cannot be easily simulated. This is particularly the case for individual resorption kinetics of the drugs, leading to different serum blood levels in vivo. Additionally, only one agent of every drug type (e.g., pantoprazole as PPI) was used while there are a variety of other drugs with the same therapeutic purpose, but with slightly different chemical structures, are available on the market. Therefore, the results must be carefully interpreted and cannot be generalized to the whole drug family when they are transferred from the bench to the bedside. Subsequent studies will be performed using a three-dimensional cell culture model to emulate the bone formation in vivo while using different concentrations of the drugs.

\section{Materials and Methods}

\subsection{Experimental Design at a Glance}

Human mesenchymal stem cells (hMSC) were harvested from the femoral bone cavity of healthy donors $(\mathrm{n}=4)$ followed by cell expansion in T-175 flasks. All donors were Caucasians, three male (age 67, 79,72) and one female (age 67). The cells were seeded in $35 \mathrm{~mm}$ flat bottom petri dishes: donor 16 -fold, donor 2 2-fold, donor 3 2-fold, donor 4 2 -fold each in ten different groups. This resulted in 12 dishes from 4 different donors for each group, and 120 dishes for the entire experiment.

Group 1 Non-osteogenic negative control group (NCG)

Group 2 Osteogenic differentiation media without and drugs (OSM)

Group 3 OSM with pantoprazole (PAN)

Group 4 OSM with ibuprofen (IBU)

Group 5 OSM with diclofenac (DIC)

Group 6 OSM with metamizole (MET)

Group 7 OSM with ibuprofen and pantoprazole (IBU + PAN)

Group 8 OSM with diclofenac and pantoprazole (DIC + PAN)

Group 9 OSM with metamizole and pantoprazole (MET + PAN)

Group 10 OSM with diclofenac, metamizole and pantoprazole (DIC + MET + PAN) 
The exact media formula for each group is described in Section 4.6 Preparation of Cell Culture Media and Drug Concentrations.

Dishes were cultured for 21 days in the appropriate cell culture media at $37^{\circ} \mathrm{C}$ and $5 \%$ $\mathrm{CO}_{2}$ with media change every two days. The cell cultures were subsequently terminated and washed and then incubated with $5 \mathrm{MBq}$ of the radioactive tracer ${ }^{99 \mathrm{~m}} \mathrm{Tc}-\mathrm{HDP}$. Gamma camera imaging/analysis and post-imaging software processing were then performed. During this analysis and processing, regions of interest were defined to calculate the exact amount of tracer bound to each dish. This reflected the amount of hydroxyapatite and hence the osteogenic potential of the cell culture media.

\section{2. hMSC Harvest and Expansion}

Bone marrow aspirates were obtained from the proximal femoral cavity of four healthy donors $(n=4)$ under general anaesthesia during an elective surgical procedure for total hip arthroplasty after informed consent and positive approval by the local ethics committee. During preparation of the proximal femoral bone cavity, $10-15 \mathrm{~mL}$ of bone marrow was collected into a $20 \mathrm{~mL}$ syringe $\left(\mathrm{BD}^{\circledR}\right)$ (San Jose, CA 95131, USA) containing $1000 \mathrm{IU}$ heparin (5000 IU/mL; B-Braun ${ }^{\circledR}$ ) (Melsungen, Germany). Individual samples were diluted 1:1 with phosphate-buffered saline (PBS; Invitrogen ${ }^{\circledR}$ ) (Thermo Fisher Scientific, Waltham, MA, USA) and washed twice with PBS. The mononuclear cell fraction was isolated by Ficoll gradient centrifugation (Ficoll-Paque-PLUS; GE Healthcare ${ }^{\circledR}$ ) (VWR International $\mathrm{GmbH}$, Darmstadt, Germany). Mononuclear cells were plated in T-175 polystyrene tissue culture flasks (BD Falcon ${ }^{\circledR}$ ) (Thermo Fisher Scientific, Waltham, MA, USA) at a density of $5 \times 10^{5} / \mathrm{cm}^{2}$ and cultured in a humidified $5 \% \mathrm{CO} 2$ atmosphere at $37{ }^{\circ} \mathrm{C}$ in low-glucose Dulbecco's modified Eagle's medium (DMEM-LG, Invitrogen ${ }^{\circledR}$ ) (Thermo Fisher Scientific, Waltham, MA, USA) containing $10 \%$ heat inactivated $\left(56{ }^{\circ} \mathrm{C}, 30 \mathrm{~min}\right.$ ) foetal bovine serum (FBS, Invitrogen ${ }^{\circledR}$ ) (Thermo Fisher Scientific, Waltham, MA, USA) and $1 \%$ Penicillin/Streptomycin (Invitrogen ${ }^{\circledR}$ ) (Thermo Fisher Scientific, Waltham, MA, USA). After $48 \mathrm{~h}$, nonadherent cells were removed and the adherent cells were washed with PBS. Media was changed every 2-3 days. At $90 \%$ confluence, cells were trypsinized. For further experiments, these $\mathrm{P} 0$ cells were frozen in liquid nitrogen in $0.5 \mathrm{~mL}$ aliquots containing $5 \times 10^{5}$ cells in DMEM-LG with $20 \%$ FBS and $10 \%$ DMSO (Sigma ${ }^{\circledR}$ St. Louis MO, USA).

\subsection{Osteogenic Differentiation Assay}

P0 human donor $(\mathrm{n}=4) \mathrm{MSC}$, frozen in N2 in $0.5 \mathrm{ml}$ aliquot vials, containing $5 \times 10^{5}$ cells each, were thawed and seeded into T-175 flask (BD Falcon ${ }^{\circledR}$ ) (Thermo Fisher Scientific, Waltham, MA, USA) with 250,000 cells each, and cultured for 10 days to harvest enough cells for the further experiments. Donor 1: 3 vials $=6$ flasks, donor 2: 1 vial $=2$ flasks, donor 3: 1 vial $=2$ flasks, and donor 4: 1 vial $=2$ flasks

During this cell expansion stage, Dulbecco's modified Eagle's medium (DMEMLG, Invitrogen $\left.{ }^{\circledR}\right)$ (Thermo Fisher Scientific, Waltham, MA, USA) containing $10 \%$ heat inactivated $\left(56^{\circ} \mathrm{C}, 30 \mathrm{~min}\right)$ foetal bovine serum (FBS, Invitrogen ${ }^{\circledR}$ Thermo Fisher Scientific, Waltham, MA, USA) and $1 \%$ Penicillin/Streptomycin (Invitrogen ${ }^{\circledR}$ (Thermo Fisher Scientific, Waltham, MA, USA) was used with media change every 2-3 days cultured in a humidified $5 \% \mathrm{CO} 2$ atmosphere at $37^{\circ} \mathrm{C}$. After 10 days, approximately $80 \%-90 \%$ of confluence was reached and cells were trypsinized and resuspended, and cells from every donor were seeded at a density of 15,000 cells $/ \mathrm{cm}^{2}$ into $35 \mathrm{~mm}$ flat bottom petri dishes (Corning ${ }^{\circledR}$ ) (Corning NY, USA).

For every group (total of 10 groups) six dishes were seeded with cells from donor 1 , two dishes were seeded with cells from donor 2, two dishes were seeded with cells from donor 3 and two dishes were seeded with cells from donor 4, resulting in a total of 120 dishes.

Immediately after seeding, the designated media was added to the dishes from each group.

Group 1 (NCG): Non-osteogenic, negative control group (NCG) 
Group 2 (OSM): Standard osteogenic media (OSM)

Group 3 (PAN): OSM with pantoprazole

Group 4 (IBU): OSM with ibuprofen

Group 5 (DIC): OSM with diclofenac

Group 6 (MET): OSM with metamizole

Group 7 (IBU + PAN): OSM with ibuprofen and pantoprazole containing

Group 8 (DIC + PAN): OSM with diclofenac and pantoprazole

Group 9 (MET + PAN): OSM with metamizole and pantoprazole

Group 10 (DIC + MET + PAN): OSM with diclofenac, metamizole and pantoprazole

Cells were treated for 21 days with specific media change every $2-3$ days, cultured in a humidified $5 \% \mathrm{CO} 2$ atmosphere at $37^{\circ} \mathrm{C}$. The exact media formula for each group is described in Section 4.6.

\section{4. ${ }^{99 m} \mathrm{Tc}-\mathrm{HDP}$ Labelling}

After termination of the cell cultures, the medium was removed and the dishes carefully washed twice with phosphate-buffered saline (PBS, Invitrogen ${ }^{\circledR}$ Thermo Fisher Scientific, Waltham, MA, USA). An aliquot of $5 \mathrm{MBq}{ }^{99 \mathrm{~m}}$ Tc-HDP (Technescan-HDP ${ }^{\circledR}$, Mallinckrodt Pharmaceuticals, Staines-upon Thames, UK)) in $2 \mathrm{~mL}$ of $0.9 \% \mathrm{NaCl}$ solution was added to each dish. Technetium activity was assayed with a dose calibrator (Activimeter ISOMED 1010, Nuklear-Medizintechnik Dresden GmbH, Dresden, Germany). Dishes were then incubated at room temperature for $2 \mathrm{~h}$. The remaining liquid ${ }^{99 \mathrm{~m}} \mathrm{Tc}-\mathrm{HDP}$ was removed and the dishes were washed three times (for approximately $30 \mathrm{~min}$ ) with $2 \mathrm{~mL}$ PBS each to remove the unbound tracer. Six blank dishes were incubated with the tracer in parallel to determine the mean amount of background uptake. The dishes were then placed directly on the detector of a gamma camera (E.CAM+, Siemens, Erlangen, Germany) with a $256 \times 256$ detector grid and the radionuclide counts were acquired for $180 \mathrm{~s}[21,29]$.

To determine the exact bound activity emitted by each dish, the acquisition software Xeleris ${ }^{\circledR} 3$ (GE Healthcare ${ }^{\circledR}$ Chalfont St Giles, Buckinghamshire, UK) was used to define regions of interest (ROIs) around each dish within the detection area. Each ROI had exactly the same pixel size. The number of gamma counts for each ROI was calculated by the software, reflecting the amount of bound tracer and therefore the amount of hydroxyapatite. After imaging, the dishes were stored at room temperature for $72 \mathrm{~h}$ to allow ${ }^{99 \mathrm{~m}} \mathrm{Tc}-\mathrm{HDP}$ to decay to background levels.

\subsection{Statistics}

SPSS Statistics ${ }^{\circledR}$ Version 20, (IBM, Armonk, NY, USA) was used for the statistical analysis. Statistical significance was set to $p \leq 0.05$. A Kolmogorov-Smirnov-Test for normal distribution was performed prior to further analysis of the data.

To reveal differences caused by the influence of the different media towards the osteogenic differentiation of the cells three one-factor ANOVA analyses with Bonferroni post-hoc testing were performed. Each ANOVA analyzed differences between the negative control group (group 1), the standard osteogenic group (group 2), the pantoprazole group (group 3), one of the standard pain drugs (group 4-6) and the standard pain drug + pantoprazole (group 7-9). PAN.

ANOVA 1: Group 1 NCG, group 2 OSM, group 3 PAN, group 4 IBU, group 7 IBU +

ANOVA 2: Group 1 NCG, Group 2 OSM, group 3 PAN, group 5 DIC, group 8 DIC + PAN.

ANOVA 3: Group 1 NCG, Group 2 OSM, group 3 PAN, group 6 MET, group 9 MET + PAN.

The fourth ANOVA was performed to analyze statistical differences between the negative control group (group 1), the standard osteogenic group (group 2), the pantoprazole group (group 3) and the group in which diclofenac was combined with metamizole and pantoprazole (group 10) 
ANOVA 4: Group 1 NCG, Group 2 OSM, group 3 PAN, group 10 DIC + MET + PAN.

\subsection{Preparation of Cell Culture Media and Drug Concentrations}

All used drugs are certified by the German authority board (Federal Institute for drugs and medical devise, BfArM) for the human application. Reference for the applied drug concentrations in the cell culture media was taken from the pharmacokinetic and pharmacodynamic data supplied by the manufacturer corresponding to the concentrations in the human body.

Group 1 (NCG): Non-osteogenic, negative control group (NCG), containing DMEMLG (Invitrogen ${ }^{\circledR}$ Thermo Fisher Scientific, Waltham, MA, USA), 10\% foetal bovine serum (FBS, Invitrogen ${ }^{\circledR}$ Thermo Fisher Scientific, Waltham, MA, USA) and 1\% Penicillin/ Streptomycin (Invitrogen ${ }^{\circledR}$ Thermo Fisher Scientific, Waltham, MA, USA).

Group 2 (OSM): OSM containing DMEM-LG (Invitrogen ${ }^{\circledR}$ Thermo Fisher Scientific, Waltham, MA, USA), 10\% foetal bovine serum (FBS, Invitrogen ${ }^{\circledR}$ Thermo Fisher Scientific, Waltham, MA, USA) and 1\% Penicillin/Streptomycin (Invitrogen ${ }^{\circledR}$ Thermo Fisher Scientific, Waltham, MA, USA) and the osteogenic supplements $100 \mathrm{nM}$ dexamethasone (Sigma ${ }^{\circledR}$ St. Louis MO, USA), $10 \mathrm{mM}$ B-glycerol phosphate (Sigma ${ }^{\circledR}$ St. Louis MO, USA), and $0.173 \mathrm{mM}$ L-ascorbic acid-2-phosphate (Wako ${ }^{\circledR}$ FUJIFILM Wako Chemicals Europe GmbH, Neuss, Germany) [30].

Group 3 (PAN): OSM with pantoprazole, containing (OSM) and $1.5 \mu \mathrm{g} / \mathrm{mL}$ pantoprazole (Pantozol ${ }^{\circledR}$, TAKEDA GmbH, Berlin, Germany) [31].

Group 4 (IBU): OSM with ibuprofen, containing (OSM) and $30 \mu \mathrm{g} / \mathrm{mL}$ ibuprofen (Ibuprofen ratiopharm ${ }^{\circledR}$, ratiopharm GmbH, Ulm, Germany) [22].

Group 5 (DIC): OSM with diclofenac containing (OSM) and $1 \mu \mathrm{g} / \mathrm{mL}$ diclofenac (Voltaren dispers ${ }^{\circledR}$, Novartis AG, Basel, Swiss) [23].

Group 6 (MET): OSM with metamizole, containing (OSM) and $17.3 \mu \mathrm{g} / \mathrm{mL}$ metamizole (Novalgin ${ }^{\circledR}$, Sanofi-Aventis GmbH, Frankfurt am Main, Germany) [32].

Group 7 (IBU + PAN): OSM with ibuprofen and pantoprazole containing (OSM) and $30 \mu \mathrm{g} / \mathrm{mL}$ ibuprofen (Ibuprofen ratiopharm ${ }^{\circledR}$, ratiopharm $\mathrm{GmbH}$ ) [22] and $1.5 \mu \mathrm{g} / \mathrm{mL}$ pantoprazole (Pantozol ${ }^{\circledR}$, TAKEDA GmbH, Berlin, Germany) [31].

Group 8 (DIC + PAN): OSM with diclofenac and pantoprazole containing (OSM), $1 \mu \mathrm{g} / \mathrm{mL}$ diclofenac (Voltaren dispers ${ }^{\circledR}$, Novartis AG, Basel, Swiss) [23] and $1.5 \mu \mathrm{g} / \mathrm{mL}$ pantoprazole (Pantozol ${ }^{\circledR}$, TAKEDA GmbH, Berlin, Germany) [31].

Group 9 (MET + PAN): OSM with metamizole and pantoprazole containing (OSM), $17.3 \mu \mathrm{g} / \mathrm{mL}$ metamizole (Novalgin ${ }^{\circledR}$, Sanofi-Aventis GmbH, Basel, Swiss) [32] and $1.5 \mu \mathrm{g} / \mathrm{mL}$ pantoprazole (Pantozol ${ }^{\circledR}$, TAKEDA GmbH, Berlin, Germany) [31].

Group 10 (DIC + MET + PAN): OSM with diclofenac, metamizole and pantoprazole containing (OSM), $1 \mu \mathrm{g} / \mathrm{mL}$ diclofenac (Voltaren dispers ${ }^{\circledR}$, Novartis AG) [23], $17.3 \mu \mathrm{g} / \mathrm{mL}$ metamizole (Novalgin ${ }^{\circledR}$, Sanofi-Aventis GmbH, Frankfurt am Main, Germany) [32] and $1.5 \mu \mathrm{g} / \mathrm{mL}$ pantoprazole (Pantozol ${ }^{\circledR}$, TAKEDA GmbH) [31].

Author Contributions: Conceptualization, T.G. (Tobias Grossner) and T.G. (Tobias Gotterbarm); methodology, T.G. (Tobias Grossner) and U.H.; software, U.H. and T.G. (Tobias Grossner); validation, T.G. (Tobias Grossner), U.H. and T.G. (Tobias Gotterbarm); formal analysis, T.G. (Tobias Grossner); investigation, T.G. (Tobias Grossner); resources, T.G. (Tobias Grossner); data curation, T.G. (Tobias Grossner); writing—original draft preparation, T.G. (Tobias Grossner); writing—review and editing, T.G. (Tobias Grossner), T.G. (Tobias Gotterbarm); visualization, T.G. (Tobias Grossner); supervision, T.G. (Tobias Gotterbarm); project administration, T.G. (Tobias Grossner); funding acquisition, n/a. All authors have read and agreed to the published version of the manuscript.

Funding: This research received no specific grant from any funding agency in the public, commercial or not-for-profit sectors. This research was funded from internal sources of the University Hospital Heidelberg. 
Institutional Review Board Statement: The study was conducted according to the guidelines of the Declaration of Helsinki, and approved by the Ethics Committee board, University Hospital Heidelberg, Faculty of medicine, Heidelberg Germany (No. S-309/2007) on the 23 November 2007.

Informed Consent Statement: Informed consent was obtained from all subjects involved in the study.

Data Availability Statement: Full dataset of all study data can be obtained upon request.

Conflicts of Interest: The authors declare no conflict of interest. The funders had no role in the design of the study; in the collection, analyses, or interpretation of data; in the writing of the manuscript, or in the decision to publish the results.

\section{Abbreviations}

$\begin{array}{ll}\text { ANOVA } & \text { Analysis of variance } \\ \text { Bq } & \text { Becquerel } \\ \text { BM } & \text { Bone marrow } \\ \text { COX-1 } & \text { Cyclooxygenase-1 } \\ \text { COX-2 } & \text { Cyclooxygenase-2 } \\ \text { DIC } & \text { diclofenac } \\ \text { hMSC } & \text { Human mesenchymal stem cells } \\ \text { IBU } & \text { ibuprofen } \\ \text { MET } & \text { metamizole } \\ \text { MeV } & \text { Mega-electron volts } \\ \text { MSC } & \text { Mesenchymal stem cells } \\ \text { NSAID } & \text { nonsteroidal anti-inflammatory drugs } \\ \text { OSM } & \text { Osteogenic treatment group } \\ \text { NCG } & \text { Non-osteogenic, negative control group } \\ \text { OSM } & \text { Standard osteogenic media } \\ \text { PAN } & \text { pantoprazole } \\ \text { PEG2 } & \text { Prostaglandine 2 } \\ \text { PPI } & \text { Protone pump inhibitor } \\ \text { ROI } & \text { Region of interest } \\ \text { WHO } & \text { World Health Organistaion } \\ & \end{array}$

\section{References}

1. Eden Sebbag, E.; Felten, R.; Sagez, F.; Sibilia1, J.; Devilliers, H.; Arnaud, L. The world-wide burden of musculoskeletal diseases: A systematic analysis of the World Health Organization Burden of Diseases Database. Ann. Rheum. Dis. 2019, 78, 844-848. [CrossRef] [PubMed]

2. Hsu, J.R.; Mir, H.; Wally, M.K.; Seymour, R.B. Clinical Practice Guidelines for Pain Management in Acute Musculoskeletal Injury. J. Orthop. Trauma. 2019, 33, e158-e182. [CrossRef]

3. Fikentscher, T.; Grifka, J.; Benditz, A. Perioperative pain therapy in orthopedics. Orthopade 2015, 44, 727-740. [CrossRef]

4. Chou, R.; Gordon, D.B.; de Leon-Casasola, O.A.; Rosenberg, J.M.; Bickler, S.; Brennan, T.; Carter, T.; Cassidy, C.L.; Chittenden, E.H.; Degenhardt, E.; et al. Management of Postoperative Pain: A Clinical Practice Guideline from the American Pain Society, the American Society of Regional Anesthesia and Pain Medicine, and the American Society of Anesthesiologists' Committee on Regional Anesthesia, Executive Committee, and Administrative Council. J. Pain 2016, 17, 131-157.

5. Freys, S.M.; Erlenwein, J.; Koppert, W.; Meißner, W.; Pogatzki-Zahn, E.; Schwenk, W.; Simanski, C. Agreement of the Professional Association of German Anesthesiologists and the Professional Association of German Surgeons for the Organization of Postoperative Pain Therapy for Surgical Patients (revised version 2019). Der Unf. 2019, 90, 648-651.

6. Hartz, C.; Hauser, W.; Schiltenwolf, M. Long-term Application of Opioids for Non-Tumor Associated Pain-Impact of the S3 Guidelines for the Field of Orthopaedic Surgery and Trauma Surgery. Z. Orthop. Unfall 2016, 154, 134-139. [PubMed]

7. Montané, E.; Vallano, A.; Aguilera, C.; Vidal, X.; Laporte, J. Analgesics for pain after traumatic or orthopaedic surgery: What is the evidence-a systematic review. Eur. J. Clin. Pharmacol. 2006, 62, 971-988. [CrossRef]

8. Firoozabadi, R.; Alton, T.; Sagi, H.C. Heterotopic Ossification in Acetabular Fracture Surgery. J. Am. Acad. Orthop. Surg. 2017, 25, $117-124$.

9. Joice, M.; Vasileiadis, G.I.; Amanatullah, D.F. Non-steroidal anti-inflammatory drugs for heterotopic ossification prophylaxis after total hip arthroplasty: A systematic review and meta-analysis. Bone Joint J 2018, 100, 915-922. [CrossRef] 
10. Kan, S.L.; Yang, B.; Ning, G.Z.; Chen, L.X.; Li, Y.L.; Gao, S.J.; Chen, X.Y.; Sun, J.C.; Feng, S.Q. Nonsteroidal Anti-inflammatory Drugs as Prophylaxis for Heterotopic Ossification after Total Hip Arthroplasty: A Systematic Review and Meta-Analysis. Medicine 2015, 94, e828. [CrossRef] [PubMed]

11. Łegosz, P.; Otworowski, M.; Sibilska, A.; Starszak, K.; Kotrych, D.; Kwapisz, A.; Synder, M. Heterotopic Ossification: A Challenging Complication of Total Hip Arthroplasty: Risk Factors, Diagnosis, Prophylaxis, and Treatment. Biomed Res. Int. 2019, 2019, 3860142. [CrossRef]

12. Zhu, X.T.; Chen, L.; Lin, J.H. Selective COX-2 inhibitor versus non-selective COX-2 inhibitor for the prevention of heterotopic ossification after total hip arthroplasty: A meta-analysis. Medicine 2018, 97, e11649. [CrossRef]

13. Rostom, A.; Dube, C.; A Wells, G.; Tugwell, P.; Welch, V.; Jolicoeur, E.; McGowan, J.; Lanas, A. Prevention of NSAID-induced gastroduodenal ulcers. Cochrane Database Syst. Rev. 2002, CD002296. [CrossRef]

14. Hoff, M.; Skovlund, E.; Skurtveit, S.; Meyer, H.; Langhammer, A.; Søgaard, A.; Syversen, U.; Forsmo, S.; Abrahamsen, B.; Schei, B. Proton pump inhibitors and fracture risk. The HUNT study, Norway. Osteoporos. Int. 2020, 31, 109-118. [CrossRef]

15. Thong, B.K.S.; Ima-Nirwana, S.; Chin, K.-Y. Proton Pump Inhibitors and Fracture Risk: A Review of Current Evidence and Mechanisms Involved. Int. J. Environ. Res. Public Health 2019, 16, 1571. [CrossRef]

16. Costa-Rodrigues, J.; Reis, S.; Teixeira, S.; Lopes, S.; Fernandes, M.H. Dose-dependent inhibitory effects of proton pump inhibitors on human osteoclastic and osteoblastic cell activity. FEBS J. 2013, 280, 5052-5064. [CrossRef] [PubMed]

17. Prause, M.; Seeliger, C.; Unger, M.; van Griensven, M.; Haug, A.T. Pantoprazole increases cell viability and function of primary human osteoblasts in vitro. Injury 2014, 45, 1156-1164. [CrossRef] [PubMed]

18. Prause, M.; Seeliger, C.; Unger, M.; Balmayor, E.R.; Van Griensven, M.; Haug, A.T. Pantoprazole decreases cell viability and function of human osteoclasts in vitro. Mediators Inflamm. 2015, 2015, 413097. [CrossRef] [PubMed]

19. Marquez-Lara, A.; Hutchinson, I.D.; Nuñez, F.; Smith, T.L.; Miller, A.N. Nonsteroidal Anti-Inflammatory Drugs and Bone-Healing: A Systematic Review of Research Quality. JBJS Rev. 2016, 4. [CrossRef]

20. Pountos, I.; Georgouli, T.; Calori, G.M.; Giannoudis, P.V. Do nonsteroidal anti-inflammatory drugs affect bone healing? A critical analysis. Sci. World J. 2012, 2012, 606404. [CrossRef]

21. Grossner, T.; Gotterbarm, T.; Gerbaudo, V.H.; Haberkorn, U.; Spector, M. (99m)Tc-Methyl-Diphosphonate Binding to Mineral Deposits in Cultures of Marrow-Derived Mesenchymal Stem Cells in Osteogenic Medium. Tissue Eng. Part C Methods 2019, 25, 49-57. [CrossRef]

22. Abukawa, H.; Phelps, M.; Jackson, P.; Smith, R.M.; Vacanti, J.P.; Kaban, L.B.; Troulis, M.J. Effect of ibuprofen on osteoblast differentiation of porcine bone marrow-derived progenitor cells. J. Oral Maxillofac. Surg. 2009, 67, 2412-2417. [CrossRef]

23. Pountos, I.; Giannoudis, P.V.; Jones, E.; English, A.; Churchman, S.; Field, S.; Ponchel, F.; Bird, H.; Emery, P.; McGonagle, D. NSAIDS inhibit in vitro MSC chondrogenesis but not osteogenesis: Implications for mechanism of bone formation inhibition in man. J. Cell. Mol. Med. 2011, 15, 525-534. [CrossRef]

24. Yoon, D.S.; Yoo, J.H.; Kim, Y.H.; Paik, S.; Han, C.D.; Lee, J.W. The effects of COX-2 inhibitor during osteogenic differentiation of bone marrow-derived human mesenchymal stem cells. Stem. Cells Dev. 2010, 19, 1523-1533. [CrossRef]

25. Almaawi, A.; Wang, H.T.; Ciobanu, O.; Rowas, S.A.; Rampersad, S.; Antoniou, J.; Mwale, F. Effect of acetaminophen and nonsteroidal anti-inflammatory drugs on gene expression of mesenchymal stem cells. Tissue Eng. Part A 2013, 19, 1039-1046. [CrossRef]

26. Oh, N.; Kim, S.; Hosoya, K.; Okumura, M. Compensatory cellular reactions to nonsteroidal anti-inflammatory drugs on osteogenic differentiation in canine bone marrow-derived mesenchymal stem cells. J. Vet. Med. Sci. 2014, 76, 629-636. [CrossRef] [PubMed]

27. Pytlik, M.; Cegieła, U.; Nowińska, B.; Folwarczna, J.; Sliwiński, L.; Kaczmarczyk-Sedlak, I.; Bolek, D.; Korzeniowska, H. Bone remodeling after administration of proton pump (H+/K+-ATPase) inhibitors and alendronate in ovariectomized rats. Acta Pol. Pharm. 2012, 69, 113-120. [PubMed]

28. Joo, M.K.; Park, J.-J.; Lee, B.J.; Kim, J.H.; Yeon, J.E.; Kim, J.S.; Byun, K.S.; Bak, Y.-T. The effect of a proton pump inhibitor on bone metabolism in ovariectomized rats. Mol. Med. Rep. 2013, 7, 1267-1272. [CrossRef]

29. Grossner, T.L.; Haberkorn, U.; Gotterbarm, T. (99m)Tc-Hydroxydiphosphonate quantification of extracellular matrix mineralization in 3D human mesenchymal stem cell cultures. Bone Joint Res. 2019, 8, 333-341. [CrossRef] [PubMed]

30. Jaiswal, N.; Haynesworth, S.E.; Caplan, A.I.; Bruder, S.P. Osteogenic differentiation of purified, culture-expanded human mesenchymal stem cells in vitro. J. Cell. Biochem. 1997, 64, 295-312. [CrossRef]

31. Takeda_GmbH. Fachinformation für Pantozol®40 mg Magensaftresistente Tabletten. 2019. Available online: https://www. fachinfo.de/suche/fi/006633 (accessed on 5 May 2020).

32. Sanofi-Aventis_GmbH. Fachinformation für Novalgin Tropfen. 2019. Available online: https://www.fachinfo.de/suche/fi/0147 10 (accessed on 5 May 2020). 